\title{
Highly Selective Asymmetric Hydrogenation Using a Three Hindered Quadrant Bisphosphine Rhodium Catalyst
}

Garrett Hoge, * He-Ping Wu, William Kissel, Derek Pflum, Derek Greene, and Jian Bao

\section{Supporting Information}

Materials and Methods. All reactions and manipulations were performed under nitrogen in standard laboratory glassware. Asymmetric hydrogenation was performed in a nitrogen filled glovebox. THF (anhydrous, 99.9\%), acetonitrile (anhydrous, 99.8\%), ether (anhydrous, 99.8\%), methyl alcohol (anhydrous, 99.8\%), and dichloromethane (anyhydrous, 99.8\%) were obtained from Aldrich Chemical Co. Bis(1,5-cyclooctadiene)rhodium (I) tetrafluoroborate was synthesized according to literature procedure. ${ }^{\mathrm{O}}$ Hydrogen gas was used from a lecture bottle supplied by Specialty Gas. Hydrogenations were performed in a Griffin-Worden pressure vessel supplied by Kimble/Kontes.

Nuclear Magnetic Resonance. $400 \mathrm{MHz}{ }^{1} \mathrm{H}$ NMR, $100 \mathrm{MHz}{ }^{13} \mathrm{C} \mathrm{NMR}$, and $162 \mathrm{MHz}{ }^{31} \mathrm{P} \mathrm{NMR}$ spectra were obtained on "Barton" - a Varian Unity+400 (Inova400 after 08/15/00) spectrometer equipped with an Auto Switchable 4-Nuclei PFG probe, two RF channels, and a SMS-100 sample changer by Zymark. Spectra were generally acquired near room temperature $\left(21^{\circ} \mathrm{C}\right)$, and standard autolock, autoshim and autogain routines were employed. Samples are usually spun at $20 \mathrm{~Hz}$ for $1 \mathrm{D}$ experiments. ${ }^{1} \mathrm{H}$ NMR spectra were acquired using 45-degree tip angle pulses, 1.0 second recycle delay, and 16 scans at a resolution of $0.25 \mathrm{~Hz} /$ point. The acquisition window was typically $8000 \mathrm{~Hz}$ from +18 to $-2 \mathrm{ppm}$ (Reference TMS at $0 \mathrm{ppm}$ ), and processing was with 0.2 $\mathrm{Hz}$ line broadening. Typical acquisition time is 80 seconds. Regular ${ }^{13} \mathrm{C}$ NMR spectra were acquired using 45-degree tip angle pulses, 2.0 second recycle delay, and 2048 scans at a resolution of $1 \mathrm{~Hz} /$ point. Spectral width was typically $25 \mathrm{KHz}$ from +235 to $-15 \mathrm{ppm}$ (Reference 
TMS at $0 \mathrm{ppm}$ ). Proton decoupling was applied continuously, and $2 \mathrm{~Hz}$ line broadening was applied during processing. Typical acquisition time is 102 minutes. ${ }^{31} \mathrm{P}$ NMR spectra were acquired using 45-degree tip angle pulses, 1.0 second recycle delay, and 64 scans at a resolution of $2 \mathrm{~Hz} /$ point. Spectral width was typically $48 \mathrm{KHz}$ from +200 to $-100 \mathrm{ppm}$ (Reference $85 \%$ Phosphoric Acid at $0 \mathrm{ppm}$ ). Proton decoupling was applied continuously, and $2 \mathrm{~Hz}$ line broadening was applied during processing. Typical acquisition time is 1.5 minutes.

Mass Spectrometry. Mass Spectrometry was performed on a Micromass Platform LC system operating under MassLynx and OpenLynx open access software. The LC was equipped with a HP1100 quaternary LC system and a Gilson 215 liquid handler as an autosampler. Data was acquired under atmospheric pressure chemical ionization with 80:20 acetonitrile/water as the solvent. Temperatures: probe was $450^{\circ} \mathrm{C}$, source was $150^{\circ} \mathrm{C}$. Corona discharge was $3500 \mathrm{~V}$ for positive ion and $3200 \mathrm{~V}$ for negative ion.

High Performance Liquid Chromatography. High Performance Liquid Chromatography (HPLC) was performed on a series 1100 Agilent Technologies instrument equipped with a manual injector, quaternary pump, and a UV detector. The LC was PC controlled using HP Chemstation Plus Software. Normal Phase chiral HPLC was performed using a Chiracel OJ column supplied by Chiral Technologies.

Gas Chromatography. Gas Chromatography (GC) was performed on a 110 volt Varian Star 3400 equipped with an FID detector with electrometer, a model 1061 packed/530 micron ID flash injector, a model 1077 split/splitless capillary injector, a relay board that monitors four 
external events, and an inboard printer/plotter. Gas chromatography was performed using $40 \mathrm{~m} \mathrm{x}$ $0.25 \mathrm{~mm}$ Chiraldex G-TA or B-TA columns supplied by Advanced Separation Technologies, Incorporated or on a $25 \mathrm{~m}$ x $0.25 \mathrm{~mm}$ Coating Chirasil-L-Val column supplied by Chrompack.

General procedure for asymmetric hydrogenation reactions. Catalyst precursor 5a $(0.01$ mmole) was dissolved in $1 \mathrm{~mL}$ degassed methanol in a Griffin-Worden pressure vessel equipped with the attachments necessary for connection to a hydrogen cylinder. The substrate (1 mmole) was dissolved in $4 \mathrm{~mL}$ of the same solvent and this was delivered to the catalyst solution via syringe. The vessel was sealed and pressurized to $50 \mathrm{psi} \mathrm{H}_{2}$. Reaction completion times were monitored via $\mathrm{H}_{2}$ gas uptake and enantiomeric excesses were monitored via chiral GC or HPLC.

Procedure for $100 \mathrm{~g}$ asymmetric hydrogenation of 8 using 5a (27,000:1 S/C). The substrate (100 g, $442 \mathrm{mmole})$ was weighed into a hydrogenation bottle in air. The hydrogenation bottle was then transferred to a glovebox $\left(\mathrm{O}_{2}<5 \mathrm{ppm}\right)$. To the substrate was added $500 \mathrm{~mL}$ degassed $\mathrm{MeOH}$ with stirring to dissolve the substrate. Catalyst precursor 5a $(9.2 \mathrm{mg})$ was then added to the substrate solution. The hydrogenation vessel was sealed and then pressurized to $50 \mathrm{psi}_{2}$ and stirred vigorously with a Teflon coated magnet. Pressure of the reaction was maintained at a constant 50 psi $\mathrm{H}_{2}$. After 40 hours $\mathrm{H}_{2}$ uptake was complete. $\mathrm{GC}$ analysis showed $>98 \%$ conversion to 9 and $98 \%$ e.e. ( $\mathrm{S}$ isomer).

Enantiomeric Excess Determinations. Enantiomeric excesses of acrylic acid derivatives were determined as follows: N-acetylalanine methyl ester (Capillary GC, Chrompack Chiral-L-Val $(25 \mathrm{~m}), 120^{\circ} \mathrm{C}$, isothermal, (R) $\mathrm{t}_{1}=10.5 \mathrm{~min} ;(\mathrm{S}) \mathrm{t}_{2}=11.0 \mathrm{~min} ; \mathbf{N}$-acetylphenylalanine methyl 
ester (HPLC, Daicel Chiralcel OJ, $1.0 \mathrm{~mL} / \mathrm{min}, 10 \%$ isopropanol/hexane, $(\mathrm{R}) \mathrm{t}_{1}=11.6 \mathrm{~min}$; (S) $\left.\mathrm{t}_{2}=17.7 \mathrm{~min}\right) ; \mathbf{N}$-acetylphenylalanine and $\mathbf{N}$-acetylalanine were converted to their corresponding methyl esters via treatment with trimethylsilyldiazomethane and their enantiomeric excesses were analyzed as described above; $\mathbf{N}$-acetylcyclohexylglycine methyl ester (Capillary GC, Chirasil-L-Val $(25 \mathrm{~m}), 145^{\circ} \mathrm{C}$ isothermal, $(\mathrm{R}) \mathrm{t}_{1}=11.3 \mathrm{~min} ;(\mathrm{S}) \mathrm{t}_{2}=12.0$ $\min )$.

The CI-1008 precursor, 9, was acidified in its methanolic hydrogenation solution with $1 \mathrm{~N}$ $\mathrm{HCl}$. The organics were extracted into $\mathrm{CH}_{2} \mathrm{Cl}_{2}$. After drying over $\mathrm{MgSO}_{4}$ the volatiles were removed invacuo. The carboxylic acid was then converted to its corresponding methyl ester via treatment with trimethylsilyldiazomethane. The methyl ester was analyzed directly (Capillary GC, Astec GTA $(30 \mathrm{~m}), 140{ }^{\circ} \mathrm{C}$, isothermal, $\left.(\mathrm{R}) \mathrm{t}_{1}=8.8 \mathrm{~min} ;(\mathrm{S}) \mathrm{t}_{2}=9.5 \mathrm{~min}\right)$.

Absolute Configurations. Absolute configurations of the products of hydrogenation were determined by comparison of the signs of optical rotation with those of literature values: (S)-NAcetylalanine methyl ester $[\alpha]_{\mathrm{D}}{ }^{20}=-91.7^{\mathrm{o}}$ (c $\left.2, \mathrm{H}_{2} \mathrm{O}\right) ;$ ii $(\mathbf{S})-\mathbf{N}$-acetylphenylalanine methyl ester $[\alpha]_{\mathrm{D}}^{20}=+16.4^{\circ}(\mathrm{c} 2, \mathrm{MeOH}) ;$ ii $(\mathbf{S})-\mathbf{N}$-acetylcyclohexylglycine methyl ester $[\alpha]_{\mathrm{D}}{ }^{20}=-4.6^{\mathrm{o}}$ $(\mathrm{c}=0.13, \mathrm{EtOH}) . \mathrm{iv}^{\mathrm{V}}$ The CI-1008 precursor, 9, was compared in terms of elution order to an authentic sample with $(\mathrm{S})$ configuration via the method for enantiomeric excess determination (above).

\section{Preparation of (rac)-tert-butylmethylphosphino-di-tert-butylphosphinomethane (4). A} solution of 3 (20.1 g, 152 mmole) in $50 \mathrm{~mL}$ THF was stirred at $0{ }^{\circ} \mathrm{C}$. To the solution was added sBuLi (104 mL, 145 mmole) while maintaining the reaction temperature below $20{ }^{\circ} \mathrm{C}$. The addition took $20 \mathrm{~min}$, after which it turned slightly cloudy and orange. The reaction was stirred 
for one hour at $0{ }^{\circ} \mathrm{C}$. The reaction solution was then transferred via cannula to a pre-cooled solution of di-tert-butylchlorophosphine $(25 \mathrm{~g}, 138 \mathrm{mmole})$ in $50 \mathrm{~mL}$ THF at $0{ }^{\circ} \mathrm{C}$. The temperature was maintained below $20^{\circ} \mathrm{C}$. The reaction turned red immediately with addition of the first drop. The total addition took $20 \mathrm{~min}$. The reaction was stirred at $0{ }^{\circ} \mathrm{C}$ for $2 \mathrm{~h}$. To this solution was then added $\mathrm{BH}_{3} \bullet \mathrm{Me}_{2} \mathrm{~S}(14.4 \mathrm{~mL}, 152 \mathrm{mmole})$ over $10 \mathrm{~min}$. while maintaining the reaction temperature $<20^{\circ} \mathrm{C}$. The reaction was stirred for $1 \mathrm{~h}$ after which it was poured onto 100 $\mathrm{g}$ ice in $100 \mathrm{~mL} 1 \mathrm{~N} \mathrm{HCl}$ and stirred for $30 \mathrm{~min}$. The aqueous solution was extracted with EtOAc $(2 \times 100 \mathrm{~mL})$ and then the combined organic layers were dried over $\mathrm{MgSO}_{4}$ and filtered. Volatiles were then removed on a rotary evaporator. The residue was recrystallized from hot heptane producing a white crystalline solid. The solid weighed $25 \mathrm{~g}(63 \%) . \mathrm{mp}=150-152{ }^{\circ} \mathrm{C} ;{ }^{1} \mathrm{H} \mathrm{NMR}$ $\left(400 \mathrm{MHz}, \mathrm{CDCl}_{3}\right) \delta 1.88(\mathrm{t}, \mathrm{J}=12 \mathrm{~Hz}, 2 \mathrm{H}), 1.56(\mathrm{~d}, \mathrm{~J}=10 \mathrm{~Hz}, 3 \mathrm{H}), 1.33(\mathrm{~d}, \mathrm{~J}=13 \mathrm{~Hz}, 9 \mathrm{H})$, $1.27(\mathrm{~d}, \mathrm{~J}=13 \mathrm{~Hz}, 9 \mathrm{H}), 1.19(\mathrm{~d}, \mathrm{~J}=13 \mathrm{~Hz}, 9 \mathrm{H}), 0.61(\mathrm{br} \mathrm{q}, 6 \mathrm{H}) ;{ }^{13} \mathrm{C}$ NMR $\left(100 \mathrm{MHz}, \mathrm{CDCl}_{3}\right) \delta$ $34.29(\mathrm{~d}, \mathrm{~J}=25 \mathrm{~Hz}), 33.41$ (d, J = 25 Hz), 30.00 (d, $25 \mathrm{~Hz}), 28.30$ (s), 27.89 (s), 25.21 (s), 9.12 $(\mathrm{dd}, \mathrm{J}=21$ and $15 \mathrm{~Hz}), 6.52(\mathrm{~d}, \mathrm{~J}=32 \mathrm{~Hz}) ;{ }^{31} \mathrm{P}$ NMR $\left(162 \mathrm{MHz}, \mathrm{CDCl}_{3}\right) \delta 49.70-48.15(\mathrm{~m})$, $33.03-31.56$ (m). Anal Calcd for $\mathrm{C}_{14} \mathrm{H}_{38} \mathrm{~B}_{2} \mathrm{P}_{2}: \mathrm{C}, 57.98 ; \mathrm{H}, 13.21$. Found: $\mathrm{C}, 57.64 ; \mathrm{H}, 13.01$.

Preparation of (R)-tert-butylmethylphosphino-di-tert-butylphosphinomethane (2a). Both 4a (290 mg, $1.0 \mathrm{mmol})$ and DABCO (135 mg, $1.2 \mathrm{mmol})$ were dissolved in $10 \mathrm{ml}$ of degassed toluene at $20^{\circ} \mathrm{C}$. The solution was stirred for $4 \mathrm{~h}$ at $80{ }^{\circ} \mathrm{C}$. The solvent was removed invacuo and then the residue was extracted with hexane $(3 \mathrm{X} 20 \mathrm{ml})$. The combined organic extracts were concentrated and dried producing 2a (228 mg, 87\%) as colorless oil. ${ }^{1} \mathrm{H}$ NMR (400 MHz, $\left.\mathrm{CDCl}_{3}\right) \delta 1.47-1.41(\mathrm{~m}, 2 \mathrm{H}), 1.09(\mathrm{~d}, \mathrm{~J}=11 \mathrm{~Hz}, 9 \mathrm{H}), 1.03(\mathrm{~d}, \mathrm{~J}=11 \mathrm{~Hz}, 9 \mathrm{H}), 0.94(\mathrm{~d}, \mathrm{~J}=11 \mathrm{~Hz}$ 9H), $0.93(\mathrm{~d}, \mathrm{~J}=3 \mathrm{~Hz}, 3 \mathrm{H}) ;{ }^{13} \mathrm{C} \mathrm{NMR}\left(100 \mathrm{MHz}, \mathrm{CDCl}_{3}\right) \delta 7.44(\mathrm{dd}, \mathrm{J}=19$ and $6 \mathrm{~Hz}), 16.09$ 
(dd, $\mathrm{J}=32$ and $25 \mathrm{~Hz}), 26.63(\mathrm{~d}, \mathrm{~J}=14 \mathrm{~Hz}), 27.95(\mathrm{dd}, \mathrm{J}=23$ and $3 \mathrm{~Hz}), 29.73(\mathrm{~d}, \mathrm{~J}=14 \mathrm{~Hz})$, $30.16(\mathrm{dd}, \mathrm{J}=13$ and $4 \mathrm{~Hz}), 31.70(\mathrm{dd}, \mathrm{J}=23$ and $9 \mathrm{~Hz}), 32.16(\mathrm{dd}, \mathrm{J}=23$ and $3 \mathrm{~Hz}) ;{ }^{31} \mathrm{P}$ NMR $\left(162 \mathrm{MHz}, \mathrm{CDCl}_{3}\right) \delta-13.66$ (br m), 18.35 (br m).

Preparation of $(+)-($ S)-tert-butylmethylphosphino-di-tert-butylphosphinomethane)-(1,5cyclooctadiene) rhodium(I) tetrafluoroborate (5a). A solution of $\mathbf{2 a}(66 \mathrm{mg}, 0.25 \mathrm{mmol})$ in 5 $\mathrm{ml}$ of THF was added dropwise to a solution of $\left[\mathrm{Rh}(\mathrm{COD})_{2}\right] \mathrm{BF}_{4}(102 \mathrm{mg}, 0.25 \mathrm{mmol})$ in $10 \mathrm{ml}$ of methanol at $20{ }^{\circ} \mathrm{C}$ with stirring. After addition, the reaction mixture was stirred for $1 \mathrm{~h}$ and solvent was removed in vacuo to provide a red solid. Recrystallization of product from warm THF provided 5a (89 mg, 64\%) as a red crystalline product. $[\alpha]^{24}{ }_{\mathrm{D}}=+52.4^{\circ}(c 0.9, \mathrm{MeOH}) ;{ }^{1} \mathrm{H}$ NMR (400 MHz, $\left.\mathrm{CDCl}_{3}\right) \delta 5.63-5.51(\mathrm{~m}, 2 \mathrm{H}), 5.11(\mathrm{br} \mathrm{s}, 2 \mathrm{H}), 3.48-3.328(\mathrm{~m}, 1 \mathrm{H}), 3.14(\mathrm{dt}, \mathrm{J}$ $=17$ and $10 \mathrm{~Hz}, 1 \mathrm{H}), 2.49-2.25(\mathrm{~m}, 4 \mathrm{H}), 2.21-2.09(\mathrm{~m}, 4 \mathrm{H}), 1.69(\mathrm{~d}, \mathrm{~J}=9 \mathrm{~Hz}, 3 \mathrm{H}), 1.39(\mathrm{~d}, \mathrm{~J}$ $=14 \mathrm{~Hz}, 9 \mathrm{H}), 1.33(\mathrm{~d}, \mathrm{~J}=14 \mathrm{~Hz}, 9 \mathrm{H}), 1.13(\mathrm{~d}, \mathrm{~J}=16 \mathrm{~Hz}, 9 \mathrm{H}) ;{ }^{13} \mathrm{C} \mathrm{NMR}\left(100 \mathrm{MHz}, \mathrm{CDCl}_{3}\right) \delta$ $100.20(\mathrm{dd}, \mathrm{J}=9$ and $6 \mathrm{~Hz}), 97.70(\mathrm{dd}, \mathrm{J}=9$ and $6 \mathrm{~Hz}), 92.95(\mathrm{t}, \mathrm{J}=8 \mathrm{~Hz}), 92.27(\mathrm{~d}, \mathrm{~J}=8 \mathrm{~Hz})$, 37.68 (m), 36.04 (d, J = 9 Hz), 32.54 (m), 31.48 (s), 30.94 (s), 30.09 (d, J = 5 Hz), 29.81 (d, J = 5 Hz), 29.32 (s), $29.16(\mathrm{~s}), 26.57$ (d, J = 5 Hz), 9.58 (d, J = 21 Hz); ${ }^{31} \mathrm{P}$ NMR (162 MHz, $\left.\mathrm{CDCl}_{3}\right) \delta$ $-3.97(\mathrm{dd}, \mathrm{J}=126$ and $56 \mathrm{~Hz}),-29.36(\mathrm{dd}, \mathrm{J}=126$ and $56 \mathrm{~Hz})$. Anal Calcd for $\mathrm{C}_{21} \mathrm{H}_{42} \mathrm{BF}_{4} \mathrm{P}_{2} \mathrm{Rh}$ : C, 46.18; H, 7.75. Found: C, 45.66; H, 7.19. 
Chiral Preparatory HPLC Method for the Separation of the Enantiomers of 4

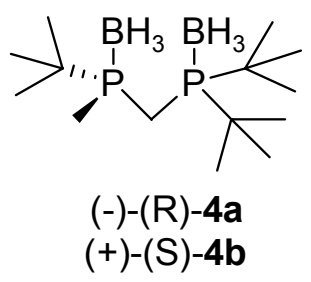

Column: $\quad$ Daicel Chiralpak AD (250x20 mm, 10 micron)

Mobile Phase: $\quad 99.25: 0.75$ [hexanes:isopropanol]

Flow Rate: $\quad 9 \mathrm{ml} / \mathrm{minute}$

Detector: $\quad$ none (* please see note below)

Concentration: $\quad 2 \mathrm{mg} / \mathrm{ml}$

Diluent: $\quad$ mobile phase

Injection Volume: $\quad 500 \mu \mathrm{L}$

Run Time: $\quad 20$ minutes

* A prep scale RI detector was not available. Therefore, no detection was used to monitor the retention times of the enantiomers. Solvent was fractionated via fraction collector and fractions were assayed using the analytical method provided.

\section{Analytical Method}

Column:

Mobile Phase:

Flow Rate:

Detector:

Column Temperature:

Concentration:

Diluent:

Injection Volume:

Run Time:
Daicel Chiralpak AD (250x4.6 mm, 10 micron)

99.25:0.75 [hexanes:isopropanol]

$1 \mathrm{ml} / \mathrm{minute}$

RI $\left(35^{\circ} \mathrm{C}\right)$

$30{ }^{\circ} \mathrm{C}$

$2 \mathrm{mg} / \mathrm{ml}$

mobile phase

$25 \mu \mathrm{L}$

13 minutes

Retention times:

$4 \mathrm{a}=6.8$ minutes; $[\alpha]^{24}{ }_{\mathrm{D}}=-5.5^{\circ}(c 0.5, \mathrm{MeOH})$;

$4 b=8.2$ minutes 


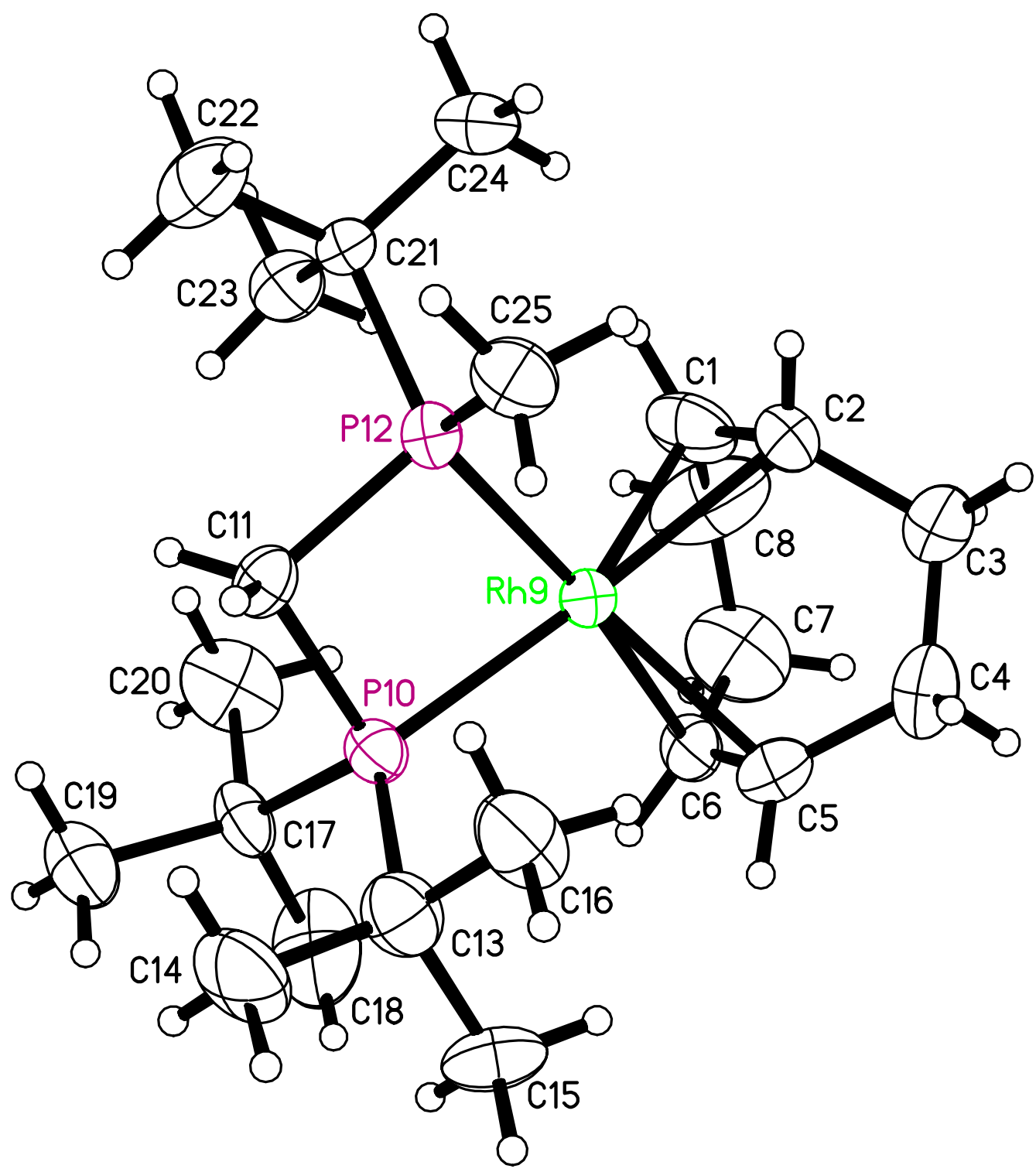

Figure 1S. ORTEP Drawing of Compound 5a. 
Table 1S. Crystal data and structure refinement for compound 5a.

Identification code

Empirical formula

Formula weight

Temperature

Wavelength

Crystal system

Space group

Unit cell dimensions

Volume

$\mathrm{Z}$

Density (calculated)

Absorption coefficient

$\mathrm{F}(000)$

Crystal size

Theta range for data collection

Index ranges

Reflections collected

Independent reflections

Completeness to theta $=26.45^{\circ}$

Absorption correction

Refinement method

Data / restraints / parameters

Goodness-of-fit on $\mathrm{F}^{2}$

Final $\mathrm{R}$ indices [I $>2 \operatorname{sigma}(\mathrm{I})]$

$\mathrm{R}$ indices (all data)

Absolute structure parameter

Extinction coefficient

Largest diff. peak and hole
AA02

$\mathrm{C}_{22} \mathrm{H}_{44} \mathrm{BF}_{4} \mathrm{P}_{2} \mathrm{Rh}$

560.23

223(2) K

$0.71073 \AA$

Monoclinic

P2(1)

$$
\begin{array}{ll}
\mathrm{a}=8.4395(4) \AA & \alpha=90^{\circ} . \\
\mathrm{b}=16.8558(7) \AA & \beta=93.1200(10)^{\circ} . \\
\mathrm{c}=18.3565(8) \AA & \gamma=90^{\circ} .
\end{array}
$$

2607.4(2) $\AA^{3}$

4

$1.427 \mathrm{Mg} / \mathrm{m}^{3}$

$0.814 \mathrm{~mm}^{-1}$

1168

$0.10 \times 0.16 \times 0.32 \mathrm{~mm}^{3}$

1.64 to $26.45^{\circ}$.

$-10<=\mathrm{h}<=10,-21<=\mathrm{k}<=21,-22<=\mathrm{l}<=22$

23133

10615 [R(int $)=0.0534]$

$99.7 \%$

\section{SADABS}

Full-matrix least-squares on $\mathrm{F}^{2}$

10615 / 1 / 542

1.020

$\mathrm{R} 1=0.0550, \mathrm{wR} 2=0.1125$

$\mathrm{R} 1=0.0670, \mathrm{wR} 2=0.1181$

$-0.04(3)$

$0.00000(17)$

0.697 and -0.569 e. $\AA^{-3}$ 
Table 2S. Atomic coordinates ( $\left.\mathrm{x} 10^{4}\right)$ and equivalent isotropic displacement parameters $\left(\AA^{2} \times 10^{3}\right)$ for compound 5a. $\mathrm{U}(\mathrm{eq})$ is defined as one third of the trace of the orthogonalized $\mathrm{U}_{\mathrm{ij}}$ tensor.

\begin{tabular}{|c|c|c|c|c|}
\hline & $\mathrm{X}$ & $\mathrm{y}$ & $\mathrm{z}$ & $\mathrm{U}(\mathrm{eq})$ \\
\hline $\mathrm{C}(1)$ & $-1951(10)$ & $2746(5)$ & $-838(4)$ & $52(2)$ \\
\hline $\mathrm{C}(2)$ & $-472(10)$ & $2966(6)$ & $-553(4)$ & $48(2)$ \\
\hline $\mathrm{C}(3)$ & $-207(14)$ & $3787(5)$ & $-243(5)$ & $71(3)$ \\
\hline $\mathrm{C}(4)$ & $265(12)$ & $4372(6)$ & $-801(5)$ & $66(3)$ \\
\hline$C(5)$ & $-395(11)$ & $4273(5)$ & $-1564(5)$ & $53(2)$ \\
\hline$C(6)$ & $-1893(10)$ & $4087(5)$ & $-1795(4)$ & $47(2)$ \\
\hline$C(7)$ & $-3237(11)$ & $3996(7)$ & $-1310(6)$ & $74(3)$ \\
\hline $\mathrm{C}(8)$ & $-3408(11)$ & $3242(6)$ & $-939(7)$ & $84(4)$ \\
\hline $\mathrm{Rh}(9)$ & $-463(1)$ & $2966(1)$ & $-1763(1)$ & $26(1)$ \\
\hline $\mathrm{P}(10)$ & $213(2)$ & $2832(1)$ & $-2975(1)$ & $28(1)$ \\
\hline $\mathrm{C}(11)$ & $833(8)$ & $1776(4)$ & $-2877(4)$ & $33(2)$ \\
\hline $\mathrm{P}(12)$ & $638(2)$ & $1737(1)$ & $-1881(1)$ & $30(1)$ \\
\hline$C(13)$ & $2022(8)$ & $3367(5)$ & $-3299(4)$ & $44(2)$ \\
\hline$C(14)$ & $2649(9)$ & $3040(7)$ & $-3987(4)$ & $62(2)$ \\
\hline$C(15)$ & $1607(11)$ & $4261(5)$ & $-3389(6)$ & $67(3)$ \\
\hline$C(16)$ & $3307(9)$ & $3306(6)$ & $-2674(4)$ & $57(2)$ \\
\hline$C(17)$ & $-1467(8)$ & $2827(4)$ & $-3693(4)$ & $36(2)$ \\
\hline$C(18)$ & $-2068(12)$ & $3666(6)$ & $-3848(5)$ & $74(3)$ \\
\hline$C(19)$ & $-1021(9)$ & $2444(5)$ & $-4409(4)$ & $51(2)$ \\
\hline$C(20)$ & $-2775(8)$ & $2343(6)$ & $-3380(5)$ & $60(3)$ \\
\hline $\mathrm{C}(21)$ & $-321(10)$ & $780(4)$ & $-1643(4)$ & $43(2)$ \\
\hline$C(22)$ & $532(11)$ & $84(5)$ & $-1986(5)$ & $58(2)$ \\
\hline$C(23)$ & $-2089(9)$ & $830(5)$ & $-1956(4)$ & $47(2)$ \\
\hline$C(24)$ & $-307(11)$ & $711(5)$ & $-821(4)$ & $55(2)$ \\
\hline$C(25)$ & $2660(9)$ & $1644(5)$ & $-1483(4)$ & $48(2)$ \\
\hline$C(51)$ & $2624(9)$ & $869(4)$ & $3172(4)$ & $37(2)$ \\
\hline$C(52)$ & 4194(9) & $675(5)$ & $3347(4)$ & $34(2)$ \\
\hline$C(53)$ & $4928(10)$ & $527(5)$ & $4091(5)$ & $50(2)$ \\
\hline$C(54)$ & $4373(10)$ & $1053(5)$ & $4677(4)$ & $52(2)$ \\
\hline$C(55)$ & 4104(9) & $1900(5)$ & $4420(4)$ & $39(2)$ \\
\hline$C(56)$ & $2676(10)$ & $2220(4)$ & $4149(4)$ & $42(2)$ \\
\hline$C(57)$ & $1148(9)$ & $1764(5)$ & $4004(5)$ & $51(2)$ \\
\hline $\mathrm{C}(58)$ & $1345(9)$ & $928(5)$ & $3713(4)$ & $46(2)$ \\
\hline $\operatorname{Rh}(59)$ & 4111(1) & $1988(1)$ & $3200(1)$ & $25(1)$ \\
\hline $\mathrm{P}(60)$ & $4834(2)$ & $2134(1)$ & 1994(1) & $26(1)$ \\
\hline$C(61)$ & $5343(8)$ & $3196(4)$ & $2103(4)$ & $34(2)$ \\
\hline$P(62)$ & $4620(2)$ & $3301(1)$ & $3020(1)$ & $29(1)$ \\
\hline$C(63)$ & $6704(8)$ & $1626(4)$ & $1732(4)$ & $37(2)$ \\
\hline$C(64)$ & $6396(11)$ & $783(6)$ & $1509(5)$ & $69(3)$ \\
\hline$C(65)$ & $7819(9)$ & $1645(6)$ & $2407(4)$ & $59(3)$ \\
\hline$C(66)$ & $7515(8)$ & $2043(7)$ & $1114(4)$ & $63(3)$ \\
\hline$C(67)$ & $3271(8)$ & $2113(5)$ & $1221(4)$ & $36(2)$ \\
\hline$C(68)$ & $1929(8)$ & $2654(5)$ & $1466(4)$ & $50(2)$ \\
\hline$C(69)$ & $3852(9)$ & $2430(5)$ & $503(4)$ & $48(2)$ \\
\hline$C(70)$ & $2585(11)$ & $1267(5)$ & $1112(5)$ & $60(3)$ \\
\hline$C(71)$ & $6048(9)$ & $3932(4)$ & $3562(4)$ & $42(2)$ \\
\hline$C(72)$ & $5483(11)$ & $4054(5)$ & $4329(4)$ & $53(2)$ \\
\hline$C(73)$ & $6193(12)$ & $4738(5)$ & $3199(5)$ & $68(3)$ \\
\hline$C(74)$ & $7672(10)$ & $3533(6)$ & $3604(5)$ & $71(3)$ \\
\hline
\end{tabular}




$\begin{array}{lccrr}\mathrm{C}(75) & 2841(9) & 3889(4) & 2943(4) & 42(2) \\ \mathrm{B}(81) & 9172(12) & 119(6) & 5472(5) & 45(2) \\ \mathrm{F}(82) & 10703(8) & 310(6) & 5521(6) & 173(5) \\ \mathrm{F}(83) & 8598(12) & 394(4) & 6075(3) & 138(3) \\ \mathrm{F}(84) & 8525(7) & 536(4) & 4894(3) & 85(2) \\ \mathrm{F}(85) & 8927(8) & -667(4) & 5410(3) & 90(2) \\ \mathrm{B}(91) & 4915(17) & 4987(11) & 492(8) & 89(5) \\ \mathrm{F}(92) & 3980(12) & 4504(5) & 207(9) & 264(9) \\ \mathrm{F}(93) & 6315(10) & 4874(9) & 378(5) & 229(7) \\ \mathrm{F}(94) & 5081(13) & 4894(5) & 1253(5) & 169(4) \\ \mathrm{F}(95) & 4392(9) & 5734(5) & 447(4) & 115(2)\end{array}$


Table 3S. Bond lengths $[\AA]$ and angles $\left[^{\circ}\right]$ for compound 5 a.

\begin{tabular}{|c|c|c|c|}
\hline $\mathrm{C}(1)-\mathrm{C}(2)$ & $1.378(11)$ & $\mathrm{C}(52)-\mathrm{Rh}(59)$ & $2.230(8)$ \\
\hline $\mathrm{C}(1)-\mathrm{C}(8)$ & $1.490(12)$ & $\mathrm{C}(53)-\mathrm{C}(54)$ & $1.489(11)$ \\
\hline $\mathrm{C}(1)-\mathrm{Rh}(9)$ & $2.198(7)$ & $\mathrm{C}(54)-\mathrm{C}(55)$ & $1.517(11)$ \\
\hline$C(2)-C(3)$ & $1.508(12)$ & $\mathrm{C}(55)-\mathrm{C}(56)$ & $1.387(10)$ \\
\hline $\mathrm{C}(2)-\mathrm{Rh}(9)$ & $2.220(7)$ & $\mathrm{C}(55)-\mathrm{Rh}(59)$ & $2.246(7)$ \\
\hline$C(3)-C(4)$ & $1.491(12)$ & $\mathrm{C}(56)-\mathrm{C}(57)$ & $1.513(11)$ \\
\hline$C(4)-C(5)$ & $1.490(12)$ & $\mathrm{C}(56)-\mathrm{Rh}(59)$ & $2.211(7)$ \\
\hline $\mathrm{C}(5)-\mathrm{C}(6)$ & $1.347(11)$ & C(57)-C(58) & $1.518(11)$ \\
\hline $\mathrm{C}(5)-\mathrm{Rh}(9)$ & $2.233(8)$ & $\mathrm{Rh}(59)-\mathrm{P}(62)$ & $2.2816(18)$ \\
\hline$C(6)-C(7)$ & $1.487(12)$ & $\mathrm{Rh}(59)-\mathrm{P}(60)$ & $2.3407(17)$ \\
\hline $\mathrm{C}(6)-\mathrm{Rh}(9)$ & $2.241(7)$ & $\mathrm{P}(60)-\mathrm{C}(61)$ & $1.850(7)$ \\
\hline $\mathrm{C}(7)-\mathrm{C}(8)$ & $1.452(13)$ & $\mathrm{P}(60)-\mathrm{C}(67)$ & $1.884(6)$ \\
\hline $\mathrm{Rh}(9)-\mathrm{P}(12)$ & $2.2867(18)$ & $\mathrm{P}(60)-\mathrm{C}(63)$ & $1.880(7)$ \\
\hline $\mathrm{Rh}(9)-\mathrm{P}(10)$ & $2.3377(18)$ & $\mathrm{C}(61)-\mathrm{P}(62)$ & $1.831(7)$ \\
\hline $\mathrm{P}(10)-\mathrm{C}(11)$ & $1.861(7)$ & $P(62)-C(75)$ & $1.799(7)$ \\
\hline $\mathrm{P}(10)-\mathrm{C}(17)$ & $1.883(6)$ & $\mathrm{P}(62)-\mathrm{C}(71)$ & $1.855(7)$ \\
\hline $\mathrm{P}(10)-\mathrm{C}(13)$ & $1.897(7)$ & $C(63)-C(64)$ & $1.497(12)$ \\
\hline C(11)-P(12) & $1.846(7)$ & $\mathrm{C}(63)-\mathrm{C}(65)$ & $1.515(10)$ \\
\hline $\mathrm{P}(12)-\mathrm{C}(25)$ & $1.827(7)$ & $\mathrm{C}(63)-\mathrm{C}(66)$ & $1.527(11)$ \\
\hline $\mathrm{P}(12)-\mathrm{C}(21)$ & $1.867(7)$ & $\mathrm{C}(67)-\mathrm{C}(68)$ & $1.540(10)$ \\
\hline C(13)-C(14) & $1.501(11)$ & $\mathrm{C}(67)-\mathrm{C}(69)$ & $1.529(10)$ \\
\hline C(13)-C(16) & $1.539(11)$ & $\mathrm{C}(67)-\mathrm{C}(70)$ & $1.547(10)$ \\
\hline$C(13)-C(15)$ & $1.554(12)$ & $\mathrm{C}(71)-\mathrm{C}(73)$ & $1.520(10)$ \\
\hline $\mathrm{C}(17)-\mathrm{C}(18)$ & $1.525(11)$ & $C(71)-C(72)$ & $1.525(10)$ \\
\hline C(17)-C(20) & $1.512(10)$ & $C(71)-C(74)$ & $1.525(11)$ \\
\hline C(17)-C(19) & $1.530(9)$ & B(81)-F(83) & $1.316(11)$ \\
\hline$C(21)-C(22)$ & $1.530(10)$ & $\mathrm{B}(81)-\mathrm{F}(82)$ & $1.330(11)$ \\
\hline$C(21)-C(24)$ & $1.514(10)$ & $\mathrm{B}(81)-\mathrm{F}(85)$ & $1.345(11)$ \\
\hline$C(21)-C(23)$ & $1.571(11)$ & $\mathrm{B}(81)-\mathrm{F}(84)$ & $1.361(10)$ \\
\hline C(51)-C(52) & $1.385(10)$ & B(91)-F(93) & $1.226(14)$ \\
\hline $\mathrm{C}(51)-\mathrm{C}(58)$ & $1.509(10)$ & $\mathrm{B}(91)-\mathrm{F}(92)$ & $1.231(18)$ \\
\hline $\mathrm{C}(51)-\mathrm{Rh}(59)$ & $2.263(7)$ & $\mathrm{B}(91)-\mathrm{F}(95)$ & $1.336(16)$ \\
\hline C(52)-C(53) & $1.489(10)$ & $\mathrm{B}(91)-\mathrm{F}(94)$ & $1.404(16)$ \\
\hline $\mathrm{C}(2)-\mathrm{C}(1)-\mathrm{C}(8)$ & $128.3(9)$ & $C(2)-R h(9)-C(5)$ & $80.7(3)$ \\
\hline $\mathrm{C}(2)-\mathrm{C}(1)-\mathrm{Rh}(9)$ & 72.7(4) & $\mathrm{C}(6)-\mathrm{Rh}(9)-\mathrm{C}(5)$ & $35.1(3)$ \\
\hline $\mathrm{C}(8)-\mathrm{C}(1)-\mathrm{Rh}(9)$ & $108.1(6)$ & $\mathrm{C}(1)-\mathrm{Rh}(9)-\mathrm{P}(12)$ & $99.9(2)$ \\
\hline C(1)-C(2)-C(3) & $120.2(9)$ & $\mathrm{C}(2)-\mathrm{Rh}(9)-\mathrm{P}(12)$ & $96.8(3)$ \\
\hline $\mathrm{C}(1)-\mathrm{C}(2)-\mathrm{Rh}(9)$ & $70.9(4)$ & $\mathrm{C}(6)-\mathrm{Rh}(9)-\mathrm{P}(12)$ & $169.4(2)$ \\
\hline$C(3)-C(2)-R h(9)$ & $111.7(6)$ & $\mathrm{C}(5)-\mathrm{Rh}(9)-\mathrm{P}(12)$ & $154.5(2)$ \\
\hline $\mathrm{C}(4)-\mathrm{C}(3)-\mathrm{C}(2)$ & $112.8(8)$ & $\mathrm{C}(1)-\mathrm{Rh}(9)-\mathrm{P}(10)$ & $153.9(2)$ \\
\hline$C(5)-C(4)-C(3)$ & $118.2(8)$ & $\mathrm{C}(2)-\mathrm{Rh}(9)-\mathrm{P}(10)$ & $164.9(2)$ \\
\hline$C(6)-C(5)-C(4)$ & 128.1(9) & $\mathrm{C}(6)-\mathrm{Rh}(9)-\mathrm{P}(10)$ & $102.5(2)$ \\
\hline $\mathrm{C}(6)-\mathrm{C}(5)-\mathrm{Rh}(9)$ & $72.8(5)$ & $\mathrm{C}(5)-\mathrm{Rh}(9)-\mathrm{P}(10)$ & $104.2(2)$ \\
\hline$C(4)-C(5)-R h(9)$ & $105.6(6)$ & $\mathrm{P}(12)-\mathrm{Rh}(9)-\mathrm{P}(10)$ & $72.55(6)$ \\
\hline$C(5)-C(6)-C(7)$ & $124.7(8)$ & C(11)-P(10)-C(17) & 105.1(3) \\
\hline $\mathrm{C}(5)-\mathrm{C}(6)-\mathrm{Rh}(9)$ & $72.2(5)$ & $\mathrm{C}(11)-\mathrm{P}(10)-\mathrm{C}(13)$ & $105.0(3)$ \\
\hline$C(7)-C(6)-R h(9)$ & 108.9(6) & C(17)-P(10)-C(13) & $111.7(4)$ \\
\hline$C(8)-C(7)-C(6)$ & $118.0(8)$ & $\mathrm{C}(11)-\mathrm{P}(10)-\mathrm{Rh}(9)$ & $94.8(2)$ \\
\hline $\mathrm{C}(7)-\mathrm{C}(8)-\mathrm{C}(1)$ & $116.6(8)$ & $\mathrm{C}(17)-\mathrm{P}(10)-\mathrm{Rh}(9)$ & $117.0(2)$ \\
\hline$C(1)-R h(9)-C(2)$ & $36.3(3)$ & $\mathrm{C}(13)-\mathrm{P}(10)-\mathrm{Rh}(9)$ & $119.7(3)$ \\
\hline $\mathrm{C}(1)-\mathrm{Rh}(9)-\mathrm{C}(6)$ & $80.4(3)$ & $\mathrm{P}(12)-\mathrm{C}(11)-\mathrm{P}(10)$ & 95.2(3) \\
\hline$C(2)-R h(9)-C(6)$ & $89.7(3)$ & $\mathrm{C}(25)-\mathrm{P}(12)-\mathrm{C}(11)$ & $105.5(3)$ \\
\hline $\mathrm{C}(1)-\mathrm{Rh}(9)-\mathrm{C}(5)$ & $93.0(3)$ & $\mathrm{C}(25)-\mathrm{P}(12)-\mathrm{C}(21)$ & $103.9(4)$ \\
\hline
\end{tabular}




$\begin{array}{lc} & \\ \mathrm{C}(11)-\mathrm{P}(12)-\mathrm{C}(21) & 109.0(3) \\ \mathrm{C}(25)-\mathrm{P}(12)-\mathrm{Rh}(9) & 114.5(3) \\ \mathrm{C}(11)-\mathrm{P}(12)-\mathrm{Rh}(9) & 96.9(2) \\ \mathrm{C}(21)-\mathrm{P}(12)-\mathrm{Rh}(9) & 125.3(3) \\ \mathrm{C}(14)-\mathrm{C}(13)-\mathrm{C}(16) & 109.6(7) \\ \mathrm{C}(14)-\mathrm{C}(13)-\mathrm{C}(15) & 110.8(8) \\ \mathrm{C}(16)-\mathrm{C}(13)-\mathrm{C}(15) & 106.9(7) \\ \mathrm{C}(14)-\mathrm{C}(13)-\mathrm{P}(10) & 114.6(6) \\ \mathrm{C}(16)-\mathrm{C}(13)-\mathrm{P}(10) & 106.3(5) \\ \mathrm{C}(15)-\mathrm{C}(13)-\mathrm{P}(10) & 108.4(5) \\ \mathrm{C}(18)-\mathrm{C}(17)-\mathrm{C}(20) & 109.2(7) \\ \mathrm{C}(18)-\mathrm{C}(17)-\mathrm{C}(19) & 109.0(7) \\ \mathrm{C}(20)-\mathrm{C}(17)-\mathrm{C}(19) & 108.5(7) \\ \mathrm{C}(18)-\mathrm{C}(17)-\mathrm{P}(10) & 110.9(5) \\ \mathrm{C}(20)-\mathrm{C}(17)-\mathrm{P}(10) & 105.9(5) \\ \mathrm{C}(19)-\mathrm{C}(17)-\mathrm{P}(10) & 113.1(5) \\ \mathrm{C}(22)-\mathrm{C}(21)-\mathrm{C}(24) & 111.9(7) \\ \mathrm{C}(22)-\mathrm{C}(21)-\mathrm{C}(23) & 110.5(6) \\ \mathrm{C}(24)-\mathrm{C}(21)-\mathrm{C}(23) & 108.9(7) \\ \mathrm{C}(22)-\mathrm{C}(21)-\mathrm{P}(12) & 110.3(6) \\ \mathrm{C}(24)-\mathrm{C}(21)-\mathrm{P}(12) & 108.6(5) \\ \mathrm{C}(23)-\mathrm{C}(21)-\mathrm{P}(12) & 106.4(5) \\ \mathrm{C}(52)-\mathrm{C}(51)-\mathrm{C}(58) & 124.9(6) \\ \mathrm{C}(52)-\mathrm{C}(51)-\mathrm{Rh}(59) & 70.7(4) \\ \mathrm{C}(58)-\mathrm{C}(51)-\mathrm{Rh}(59) & 110.2(5) \\ \mathrm{C}(51)-\mathrm{C}(52)-\mathrm{C}(53) & 126.6(7) \\ \mathrm{C}(51)-\mathrm{C}(52)-\mathrm{Rh}(59) & 73.4(4) \\ \mathrm{C}(53)-\mathrm{C}(52)-\mathrm{Rh}(59) & 106.6(5) \\ \mathrm{C}(52)-\mathrm{C}(53)-\mathrm{C}(54) & 115.6(7) \\ \mathrm{C}(53)-\mathrm{C}(54)-\mathrm{C}(55) & 112.5(7) \\ \mathrm{C}(56)-\mathrm{C}(55)-\mathrm{C}(54) & 126.1(7) \\ \mathrm{C}(56)-\mathrm{C}(55)-\mathrm{Rh}(59) & 70.5(4) \\ \mathrm{C}(54)-\mathrm{C}(55)-\mathrm{Rh}(59) & 111.3(5) \\ \mathrm{C}(55)-\mathrm{C}(56)-\mathrm{C}(57) & 125.5(8) \\ \mathrm{C}(55)-\mathrm{C}(56)-\mathrm{Rh}(59) & 73.2(4) \\ \mathrm{C}(57)-\mathrm{C}(56)-\mathrm{Rh}(59) & 105.6(5) \\ \mathrm{C}(56)-\mathrm{C}(57)-\mathrm{C}(58) & 115.1(6) \\ \mathrm{C}(51)-\mathrm{C}(58)-\mathrm{C}(57) & 112.9(6) \\ \mathrm{C}(56)-\mathrm{Rh}(59)-\mathrm{C}(52) & 95.5(3) \\ \mathrm{C}(56)-\mathrm{Rh}(59)-\mathrm{C}(55) & 36.3(3) \\ \mathrm{C}(52)-\mathrm{Rh}(59)-\mathrm{C}(55) & 79.4(3) \\ \mathrm{C}(56)-\mathrm{Rh}(59)-\mathrm{C}(51) & 80.7(3) \\ \mathrm{C}(52)-\mathrm{Rh}(59)-\mathrm{C}(51) & 35.9(2) \\ \mathrm{C}(55)-\mathrm{Rh}(59)-\mathrm{C}(51) & 86.3(3) \\ \mathrm{C}(56)-\mathrm{Rh}(59)-\mathrm{P}(62) & 93.3(2) \\ \mathrm{C}(52)-\mathrm{Rh}(59)-\mathrm{P}(62) & 167.16(19) \\ \mathrm{C}(55)-\mathrm{Rh}(59)-\mathrm{P}(62) & 102.6(2) \\ \mathrm{C}(51)-\mathrm{Rh}(59)-\mathrm{P}(62) & 155.95(19) \\ \mathrm{C}(56)-\mathrm{Rh}(59)-\mathrm{P}(60) & 155.4(2) \\ \mathrm{C}(52)-\mathrm{Rh}(59)-\mathrm{P}(60) & 102.19(18) \\ \mathrm{C}(55)-\mathrm{Rh}(59)-\mathrm{P}(60) & 164.83(18) \\ \mathrm{C}(51)-\mathrm{Rh}(59)-\mathrm{P}(60) & 103.86(18) \\ \mathrm{P}(62)-\mathrm{Rh}(59)-\mathrm{P}(60) & \\ \mathrm{C}(61)-\mathrm{P}(60)-\mathrm{C}(67) & \\ \mathrm{C}(61)-\mathrm{P}(60)-\mathrm{C}(63) & \\ \mathrm{C}(67)-\mathrm{P}(60)-\mathrm{C}(63) & \\ & \end{array}$

$\begin{array}{lr}\mathrm{C}(61)-\mathrm{P}(60)-\mathrm{Rh}(59) & 94.1(2) \\ \mathrm{C}(67)-\mathrm{P}(60)-\mathrm{Rh}(59) & 120.1(2) \\ \mathrm{C}(63)-\mathrm{P}(60)-\mathrm{Rh}(59) & 117.4(2) \\ \mathrm{P}(62)-\mathrm{C}(61)-\mathrm{P}(60) & 96.0(3) \\ \mathrm{C}(75)-\mathrm{P}(62)-\mathrm{C}(61) & 107.4(3) \\ \mathrm{C}(75)-\mathrm{P}(62)-\mathrm{C}(71) & 103.9(4) \\ \mathrm{C}(61)-\mathrm{P}(62)-\mathrm{C}(71) & 108.0(3) \\ \mathrm{C}(75)-\mathrm{P}(62)-\mathrm{Rh}(59) & 112.6(3) \\ \mathrm{C}(61)-\mathrm{P}(62)-\mathrm{Rh}(59) & 96.6(2) \\ \mathrm{C}(71)-\mathrm{P}(62)-\mathrm{Rh}(59) & 127.0(2) \\ \mathrm{C}(64)-\mathrm{C}(63)-\mathrm{C}(65) & 109.5(7) \\ \mathrm{C}(64)-\mathrm{C}(63)-\mathrm{C}(66) & 108.2(7) \\ \mathrm{C}(65)-\mathrm{C}(63)-\mathrm{C}(66) & 108.3(7) \\ \mathrm{C}(64)-\mathrm{C}(63)-\mathrm{P}(60) & 111.5(6) \\ \mathrm{C}(65)-\mathrm{C}(63)-\mathrm{P}(60) & 105.9(5) \\ \mathrm{C}(66)-\mathrm{C}(63)-\mathrm{P}(60) & 113.4(5) \\ \mathrm{C}(68)-\mathrm{C}(67)-\mathrm{C}(69) & 108.6(6) \\ \mathrm{C}(68)-\mathrm{C}(67)-\mathrm{C}(70) & 107.9(6) \\ \mathrm{C}(69)-\mathrm{C}(67)-\mathrm{C}(70) & 110.3(6) \\ \mathrm{C}(68)-\mathrm{C}(67)-\mathrm{P}(60) & 105.4(5) \\ \mathrm{C}(69)-\mathrm{C}(67)-\mathrm{P}(60) & 113.5(5) \\ \mathrm{C}(70)-\mathrm{C}(67)-\mathrm{P}(60) & 110.9(5) \\ \mathrm{C}(73)-\mathrm{C}(71)-\mathrm{C}(72) & 108.7(7) \\ \mathrm{C}(73)-\mathrm{C}(71)-\mathrm{C}(74) & 108.9(7) \\ \mathrm{C}(72)-\mathrm{C}(71)-\mathrm{C}(74) & 109.8(7) \\ \mathrm{C}(73)-\mathrm{C}(71)-\mathrm{P}(62) & 110.1(5) \\ \mathrm{C}(72)-\mathrm{C}(71)-\mathrm{P}(62) & 110.2(5) \\ \mathrm{C}(74)-\mathrm{C}(71)-\mathrm{P}(62) & 109.2(5) \\ \mathrm{F}(83)-\mathrm{B}(81)-\mathrm{F}(82) & 105.0(9) \\ \mathrm{F}(83)-\mathrm{B}(81)-\mathrm{F}(85) & 110.8(8) \\ \mathrm{F}(82)-\mathrm{B}(81)-\mathrm{F}(85) & 112.9(9) \\ \mathrm{F}(83)-\mathrm{B}(81)-\mathrm{F}(84) & 108.9(9) \\ \mathrm{F}(82)-\mathrm{B}(81)-\mathrm{F}(84) & 105.9(8) \\ \mathrm{F}(85)-\mathrm{B}(81)-\mathrm{F}(84) & 112.9(8) \\ \mathrm{F}(93)-\mathrm{B}(91)-\mathrm{F}(92) & 115.2(15) \\ \mathrm{F}(93)-\mathrm{B}(91)-\mathrm{F}(95) & 117.0(18) \\ \mathrm{F}(92)-\mathrm{B}(91)-\mathrm{F}(95) & 113.3(12) \\ \mathrm{F}(93)-\mathrm{B}(91)-\mathrm{F}(94) & 96.2(12) \\ \mathrm{F}(92)-\mathrm{B}(91)-\mathrm{F}(94) & 112.1(17) \\ \mathrm{F}(95)-\mathrm{B}(91)-\mathrm{F}(94) & 100.5(11) \\ & \end{array}$


Table 4S. Anisotropic displacement parameters $\left(\AA^{2} \times 10^{3}\right)$ for compound 5a. The anisotropic displacement factor exponent takes the form: $-2 \pi^{2}\left[h^{2} a^{* 2} U_{11}+\ldots+2 h k a^{*} b^{*} U_{12}\right]$

\begin{tabular}{|c|c|c|c|c|c|c|}
\hline & $\mathrm{U}_{11}$ & $\mathrm{U}_{22}$ & $\mathrm{U}_{33}$ & $\mathrm{U}_{23}$ & $\mathrm{U}_{13}$ & $\mathrm{U}_{12}$ \\
\hline $\bar{C}(1)$ & $64(5)$ & $60(7)$ & $35(5)$ & $11(4)$ & $26(4)$ & $-3(4)$ \\
\hline$C(2)$ & $84(6)$ & $38(4)$ & $24(4)$ & $3(4)$ & $9(4)$ & $-9(5)$ \\
\hline$C(3)$ & 130(9) & $43(5)$ & $40(5)$ & $-3(4)$ & $-16(5)$ & $-17(5)$ \\
\hline$C(4)$ & $97(8)$ & $55(6)$ & $43(6)$ & $-12(5)$ & $-8(5)$ & $-16(5)$ \\
\hline$C(5)$ & $80(7)$ & $23(5)$ & $55(6)$ & $1(4)$ & $5(5)$ & $-5(4)$ \\
\hline$C(6)$ & $72(6)$ & $29(4)$ & $39(5)$ & $-8(4)$ & $-7(4)$ & $20(4)$ \\
\hline$C(7)$ & $57(6)$ & $88(8)$ & $76(8)$ & $8(6)$ & $-1(5)$ & $30(5)$ \\
\hline $\mathrm{C}(8)$ & $63(6)$ & $70(8)$ & $124(10)$ & $7(7)$ & $54(6)$ & $12(5)$ \\
\hline $\operatorname{Rh}(9)$ & $34(1)$ & $24(1)$ & 21(1) & $-1(1)$ & $3(1)$ & $0(1)$ \\
\hline $\mathrm{P}(10)$ & $32(1)$ & $30(1)$ & $23(1)$ & $2(1)$ & $4(1)$ & $2(1)$ \\
\hline $\mathrm{C}(11)$ & $38(4)$ & $34(4)$ & $28(4)$ & $-8(3)$ & $5(3)$ & $9(3)$ \\
\hline $\mathrm{P}(12)$ & $39(1)$ & $28(1)$ & $23(1)$ & $-1(1)$ & $-1(1)$ & $-2(1)$ \\
\hline $\mathrm{C}(13)$ & $38(4)$ & $53(5)$ & $41(5)$ & $5(4)$ & $10(3)$ & $-7(3)$ \\
\hline$C(14)$ & $59(5)$ & $88(7)$ & $43(5)$ & $5(5)$ & $28(4)$ & $-1(5)$ \\
\hline$C(15)$ & $79(7)$ & $44(6)$ & $78(7)$ & $23(5)$ & $20(5)$ & $-24(5)$ \\
\hline$C(16)$ & $43(5)$ & $78(7)$ & $51(5)$ & $0(5)$ & $2(4)$ & $-18(4)$ \\
\hline $\mathrm{C}(17)$ & $44(4)$ & $38(5)$ & $24(4)$ & $-9(3)$ & $-6(3)$ & $16(3)$ \\
\hline $\mathrm{C}(18)$ & $89(7)$ & 71(7) & $60(7)$ & $-11(5)$ & $-25(5)$ & $39(6)$ \\
\hline$C(19)$ & $65(5)$ & $66(6)$ & $23(4)$ & $-5(4)$ & $2(4)$ & $7(4)$ \\
\hline$C(20)$ & $29(4)$ & $86(7)$ & $65(6)$ & $8(5)$ & $-5(4)$ & $-1(4)$ \\
\hline $\mathrm{C}(21)$ & $80(6)$ & $20(3)$ & $29(4)$ & $5(3)$ & $-4(4)$ & $-5(4)$ \\
\hline $\mathrm{C}(22)$ & $78(6)$ & $28(4)$ & $69(6)$ & $-4(4)$ & $16(5)$ & $3(4)$ \\
\hline$C(23)$ & $58(5)$ & $43(5)$ & $41(5)$ & $3(4)$ & $3(4)$ & $-18(4)$ \\
\hline $\mathrm{C}(24)$ & $89(6)$ & $37(5)$ & $38(5)$ & $11(4)$ & $-9(4)$ & $-8(4)$ \\
\hline$C(25)$ & $55(5)$ & $47(5)$ & $41(5)$ & $6(4)$ & $-8(4)$ & $0(4)$ \\
\hline $\mathrm{C}(51)$ & $52(5)$ & $34(4)$ & $26(4)$ & $-11(3)$ & $3(3)$ & $-7(3)$ \\
\hline $\mathrm{C}(52)$ & $60(5)$ & $20(4)$ & $26(4)$ & $4(3)$ & $22(3)$ & $7(3)$ \\
\hline$C(53)$ & $61(5)$ & $34(5)$ & $54(6)$ & $12(4)$ & $1(4)$ & $11(4)$ \\
\hline$C(54)$ & $66(5)$ & $47(5)$ & $41(5)$ & $18(4)$ & $-3(4)$ & $8(4)$ \\
\hline $\mathrm{C}(55)$ & $58(4)$ & $36(5)$ & $24(4)$ & $1(4)$ & $3(3)$ & $-14(4)$ \\
\hline$C(56)$ & $76(6)$ & $29(5)$ & $24(4)$ & $5(3)$ & $24(4)$ & $6(3)$ \\
\hline $\mathrm{C}(57)$ & $48(5)$ & $47(6)$ & $60(5)$ & $4(4)$ & $27(4)$ & $9(4)$ \\
\hline $\mathrm{C}(58)$ & $47(4)$ & $52(5)$ & $39(5)$ & $4(4)$ & $8(3)$ & $-12(4)$ \\
\hline $\operatorname{Rh}(59)$ & $32(1)$ & $23(1)$ & 21(1) & $0(1)$ & $4(1)$ & $0(1)$ \\
\hline $\mathrm{P}(60)$ & $27(1)$ & $27(1)$ & $24(1)$ & $-2(1)$ & $3(1)$ & $-1(1)$ \\
\hline $\mathrm{C}(61)$ & $46(4)$ & $30(4)$ & $25(4)$ & $-1(3)$ & $-1(3)$ & $-8(3)$ \\
\hline $\mathrm{P}(62)$ & $40(1)$ & $25(1)$ & $22(1)$ & 1(1) & $-1(1)$ & $-2(1)$ \\
\hline $\mathrm{C}(63)$ & $37(4)$ & $43(5)$ & $32(4)$ & $-7(3)$ & $11(3)$ & $4(3)$ \\
\hline$C(64)$ & $72(6)$ & $60(6)$ & $76(7)$ & $-10(5)$ & $18(5)$ & $17(5)$ \\
\hline $\mathrm{C}(65)$ & $45(5)$ & $75(7)$ & $54(5)$ & $-7(5)$ & $-6(4)$ & $29(4)$ \\
\hline $\mathrm{C}(66)$ & 41(4) & $100(8)$ & $50(5)$ & $0(6)$ & $14(3)$ & $17(5)$ \\
\hline $\mathrm{C}(67)$ & $39(4)$ & $39(5)$ & $28(4)$ & $-2(3)$ & $-4(3)$ & $0(3)$ \\
\hline$C(68)$ & $35(4)$ & $68(6)$ & $46(5)$ & 2(4) & $-10(3)$ & $8(4)$ \\
\hline $\mathrm{C}(69)$ & $49(5)$ & $63(5)$ & $30(4)$ & $7(4)$ & $-6(3)$ & $-1(4)$ \\
\hline$C(70)$ & 91(7) & $45(5)$ & $41(5)$ & $-6(4)$ & $-25(5)$ & $-19(5)$ \\
\hline$C(71)$ & $61(5)$ & $31(4)$ & $33(4)$ & $-3(3)$ & $-3(3)$ & $-20(3)$ \\
\hline$C(72)$ & $90(7)$ & $36(5)$ & $33(4)$ & $-4(3)$ & $-7(4)$ & $-12(4)$ \\
\hline $\mathrm{C}(73)$ & $112(8)$ & $41(5)$ & $50(6)$ & $3(4)$ & $-3(5)$ & $-32(5)$ \\
\hline$C(74)$ & $69(6)$ & $73(7)$ & $70(7)$ & $-21(5)$ & $-20(5)$ & $-6(5)$ \\
\hline$C(75)$ & $65(5)$ & $26(4)$ & $35(4)$ & 1(3) & $3(4)$ & $19(3)$ \\
\hline B(81) & $59(6)$ & $43(6)$ & $34(5)$ & $-2(4)$ & $3(4)$ & $-3(4)$ \\
\hline
\end{tabular}




$\begin{array}{lcccccc}\mathrm{F}(82) & 56(4) & 221(10) & 239(11) & 125(8) & -19(5) & -26(5) \\ \mathrm{F}(83) & 299(10) & 54(4) & 71(4) & -29(3) & 100(5) & -45(5) \\ \mathrm{F}(84) & 91(4) & 94(5) & 67(4) & 15(3) & -27(3) & 0(3) \\ \mathrm{F}(85) & 143(6) & 60(4) & 66(4) & -19(3) & 7(4) & 2(4) \\ \mathrm{B}(91) & 76(9) & 113(13) & 76(10) & -11(9) & 1(8) & 49(9) \\ \mathrm{F}(92) & 127(7) & 61(5) & 580(20) & -53(9) & -154(11) & -8(5) \\ \mathrm{F}(93) & 94(6) & 435(18) & 160(9) & -181(11) & 14(6) & 21(9) \\ \mathrm{F}(94) & 270(12) & 109(7) & 134(8) & 65(6) & 56(7) & 84(7) \\ \mathrm{F}(95) & 134(6) & 111(6) & 99(6) & 35(5) & 2(4) & -4(5) \\ \end{array}$


Table 5S. Hydrogen coordinates ( $\left.\times 10^{4}\right)$ and isotropic displacement parameters $\left(\AA^{2} \times 10^{3}\right)$ for compound $\mathbf{5 a}$.

\begin{tabular}{|c|c|c|c|c|}
\hline & $\mathrm{x}$ & $\mathrm{y}$ & $\mathrm{z}$ & $\mathrm{U}(\mathrm{eq})$ \\
\hline $\mathrm{H}(1 \mathrm{~A})$ & -2184 & 2176 & -766 & 80 \\
\hline $\mathrm{H}(2 \mathrm{~A})$ & 156 & 2539 & -305 & 80 \\
\hline $\mathrm{H}(3 \mathrm{~A})$ & -1185 & 3969 & -32 & 80 \\
\hline $\mathrm{H}(3 \mathrm{~B})$ & 624 & 3763 & 151 & 80 \\
\hline $\mathrm{H}(4 \mathrm{~A})$ & -34 & 4901 & -634 & 80 \\
\hline $\mathrm{H}(4 \mathrm{~B})$ & 1425 & 4362 & -810 & 80 \\
\hline $\mathrm{H}(5 \mathrm{~A})$ & 191 & 4573 & -1928 & 80 \\
\hline $\mathrm{H}(6 \mathrm{~A})$ & -2205 & 4277 & -2292 & 80 \\
\hline $\mathrm{H}(7 \mathrm{~A})$ & -3140 & 4412 & -938 & 80 \\
\hline $\mathrm{H}(7 \mathrm{~B})$ & -4222 & 4097 & -1603 & 80 \\
\hline $\mathrm{H}(8 \mathrm{~A})$ & -4219 & 2931 & -1214 & 80 \\
\hline $\mathrm{H}(8 \mathrm{~B})$ & -3806 & 3349 & -457 & 80 \\
\hline $\mathrm{H}(11 \mathrm{~A})$ & 107 & 1412 & -3144 & 80 \\
\hline $\mathrm{H}(11 \mathrm{~B})$ & 1925 & 1686 & -3015 & 80 \\
\hline $\mathrm{H}(14 \mathrm{~A})$ & 3578 & 3339 & -4111 & 80 \\
\hline $\mathrm{H}(14 \mathrm{~B})$ & 1839 & 3081 & -4380 & 80 \\
\hline $\mathrm{H}(14 \mathrm{C})$ & 2936 & 2487 & -3913 & 80 \\
\hline $\mathrm{H}(15 \mathrm{~A})$ & 2525 & 4546 & -3550 & 80 \\
\hline $\mathrm{H}(15 \mathrm{~B})$ & 1309 & 4475 & -2926 & 80 \\
\hline $\mathrm{H}(15 \mathrm{C})$ & 730 & 4322 & -3749 & 80 \\
\hline $\mathrm{H}(16 \mathrm{~A})$ & 4262 & 3573 & -2814 & 80 \\
\hline $\mathrm{H}(16 \mathrm{~B})$ & 3542 & 2752 & -2575 & 80 \\
\hline $\mathrm{H}(16 \mathrm{C})$ & 2929 & 3554 & -2239 & 80 \\
\hline $\mathrm{H}(18 \mathrm{~A})$ & -2934 & 3649 & -4217 & 80 \\
\hline $\mathrm{H}(18 \mathrm{~B})$ & -1212 & 3988 & -4022 & 80 \\
\hline $\mathrm{H}(18 \mathrm{C})$ & -2439 & 3896 & -3404 & 80 \\
\hline $\mathrm{H}(19 \mathrm{~A})$ & -1929 & 2456 & -4757 & 80 \\
\hline H(19B) & -703 & 1898 & -4320 & 80 \\
\hline $\mathrm{H}(19 \mathrm{C})$ & -149 & 2734 & -4606 & 80 \\
\hline $\mathrm{H}(20 \mathrm{~A})$ & -3680 & 2322 & -3728 & 80 \\
\hline $\mathrm{H}(20 \mathrm{~B})$ & -3090 & 2587 & -2931 & 80 \\
\hline $\mathrm{H}(20 \mathrm{C})$ & -2396 & 1809 & -3278 & 80 \\
\hline $\mathrm{H}(22 \mathrm{~A})$ & 22 & -409 & -1860 & 80 \\
\hline $\mathrm{H}(22 \mathrm{~B})$ & 1633 & 75 & -1804 & 80 \\
\hline $\mathrm{H}(22 \mathrm{C})$ & 484 & 145 & -2512 & 80 \\
\hline $\mathrm{H}(23 \mathrm{~A})$ & -2637 & 341 & -1849 & 80 \\
\hline $\mathrm{H}(23 \mathrm{~B})$ & -2108 & 909 & -2480 & 80 \\
\hline $\mathrm{H}(23 \mathrm{C})$ & -2614 & 1272 & -1731 & 80 \\
\hline $\mathrm{H}(24 \mathrm{~A})$ & -797 & 214 & -689 & 80 \\
\hline $\mathrm{H}(24 \mathrm{~B})$ & -894 & 1150 & -626 & 80 \\
\hline $\mathrm{H}(24 \mathrm{C})$ & 780 & 725 & -620 & 80 \\
\hline $\mathrm{H}(25 \mathrm{~A})$ & 3040 & 1108 & -1554 & 80 \\
\hline $\mathrm{H}(25 \mathrm{~B})$ & 2670 & 1759 & -964 & 80 \\
\hline $\mathrm{H}(25 \mathrm{C})$ & 3345 & 2017 & -1717 & 80 \\
\hline $\mathrm{H}(51 \mathrm{~A})$ & 2237 & 703 & 2677 & 80 \\
\hline $\mathrm{H}(52 \mathrm{~A})$ & 4719 & 390 & 2955 & 80 \\
\hline $\mathrm{H}(53 \mathrm{~A})$ & 6080 & 587 & 4073 & 80 \\
\hline $\mathrm{H}(53 \mathrm{~B})$ & 4718 & -24 & 4224 & 80 \\
\hline $\mathrm{H}(54 \mathrm{~A})$ & 5162 & 1050 & 5089 & 80 \\
\hline $\mathrm{H}(54 \mathrm{~B})$ & 3379 & 841 & 4850 & 80 \\
\hline
\end{tabular}




\begin{tabular}{|c|c|c|c|c|}
\hline $\mathrm{H}(55 \mathrm{~A})$ & 4808 & 2290 & 4679 & 80 \\
\hline $\mathrm{H}(56 \mathrm{~A})$ & 2532 & 2789 & 4262 & 80 \\
\hline $\mathrm{H}(57 \mathrm{~A})$ & 468 & 2063 & 3652 & 80 \\
\hline $\mathrm{H}(57 \mathrm{~B})$ & 595 & 1733 & 4459 & 80 \\
\hline $\mathrm{H}(58 \mathrm{~A})$ & 1606 & 570 & 4123 & 80 \\
\hline $\mathrm{H}(58 \mathrm{~B})$ & 336 & 752 & 3478 & 80 \\
\hline $\mathrm{H}(61 \mathrm{~A})$ & 6486 & 3294 & 2090 & 80 \\
\hline $\mathrm{H}(61 \mathrm{~B})$ & 4763 & 3533 & 1744 & 80 \\
\hline $\mathrm{H}(64 \mathrm{~A})$ & 7380 & 539 & 1376 & 80 \\
\hline $\mathrm{H}(64 \mathrm{~B})$ & 5634 & 770 & 1095 & 80 \\
\hline $\mathrm{H}(64 \mathrm{C})$ & 5976 & 494 & 1913 & 80 \\
\hline $\mathrm{H}(65 \mathrm{~A})$ & 8807 & 1384 & 2304 & 80 \\
\hline $\mathrm{H}(65 \mathrm{~B})$ & 7334 & 1372 & 2803 & 80 \\
\hline $\mathrm{H}(65 \mathrm{C})$ & 8032 & 2192 & 2546 & 80 \\
\hline $\mathrm{H}(66 \mathrm{~A})$ & 8458 & 1750 & 999 & 80 \\
\hline $\mathrm{H}(66 \mathrm{~B})$ & 7810 & 2577 & 1265 & 80 \\
\hline $\mathrm{H}(66 \mathrm{C})$ & 6791 & 2067 & 686 & 80 \\
\hline $\mathrm{H}(68 \mathrm{~A})$ & 1077 & 2667 & 1089 & 80 \\
\hline $\mathrm{H}(68 \mathrm{~B})$ & 2336 & 3186 & 1548 & 80 \\
\hline $\mathrm{H}(68 \mathrm{C})$ & 1527 & 2449 & 1914 & 80 \\
\hline $\mathrm{H}(69 \mathrm{~A})$ & 3002 & 2396 & 127 & 80 \\
\hline $\mathrm{H}(69 \mathrm{~B})$ & 4749 & 2117 & 360 & 80 \\
\hline $\mathrm{H}(69 \mathrm{C})$ & 4175 & 2980 & 565 & 80 \\
\hline $\mathrm{H}(70 \mathrm{~A})$ & 1791 & 1269 & 710 & 80 \\
\hline $\mathrm{H}(70 \mathrm{~B})$ & 2103 & 1098 & 1554 & 80 \\
\hline $\mathrm{H}(70 \mathrm{C})$ & 3432 & 904 & 1004 & 80 \\
\hline $\mathrm{H}(72 \mathrm{~A})$ & 6242 & 4381 & 4608 & 80 \\
\hline $\mathrm{H}(72 \mathrm{~B})$ & 5391 & 3544 & 4567 & 80 \\
\hline $\mathrm{H}(72 \mathrm{C})$ & 4457 & 4315 & 4300 & 80 \\
\hline $\mathrm{H}(73 \mathrm{~A})$ & 6936 & 5064 & 3488 & 80 \\
\hline $\mathrm{H}(73 \mathrm{~B})$ & 5162 & 4994 & 3165 & 80 \\
\hline $\mathrm{H}(73 \mathrm{C})$ & 6572 & 4669 & 2714 & 80 \\
\hline $\mathrm{H}(74 \mathrm{~A})$ & 8418 & 3858 & 3892 & 80 \\
\hline $\mathrm{H}(74 \mathrm{~B})$ & 8040 & 3471 & 3115 & 80 \\
\hline $\mathrm{H}(74 \mathrm{C})$ & 7589 & 3015 & 3830 & 80 \\
\hline $\mathrm{H}(75 \mathrm{~A})$ & 3118 & 4441 & 2868 & 80 \\
\hline $\mathrm{H}(75 \mathrm{~B})$ & 2277 & 3841 & 3387 & 80 \\
\hline $\mathrm{H}(75 \mathrm{C})$ & 2170 & 3704 & 2532 & 80 \\
\hline
\end{tabular}


${ }^{\text {i }}$ Schenk, T. G.; Downes, J. M.; Milne, C. R. C.; Mackenzie, P. B.; Boucher, H. Inorg. Chem. 1985, 24, 2334.

${ }^{i i}$ Wolf, J.P., III; Neimann, C. Biochemistry, 1963, 2, 493.

iii Vineyard, B.D.; Knowles, W.S.; Sabacky, M.J.; Bachman, G.L.; Weinkauff, D.J. J. Am. Chem. Soc., 1977, 99, 5946.

${ }^{\text {iv }}$ Burk, M. J.; Gross, M. F.; Martinez, J. P. J. Am. Chem. Soc. 1995, 117, 9375. 